\title{
Prosody and quantifier semantics in Greek
}

\author{
Antonis Botinis ${ }^{1}$, Aikaterini Bakakou-Orphanou ${ }^{2}$, Anthi Chaida ${ }^{1}$ \\ ${ }_{2}^{1}$ Lab of Phonetics \& Computational Linguistics, University of Athens, Greece \\ ${ }^{2}$ Department of Linguistics, University of Athens, Greece \\ https://doi.org/10.36505/ExLing-2011/04/0009/000178
}

\begin{abstract}
This study investigates prosody and semantic specification in Greek with reference to affirmative and negative sentence quantifier functions. A production and a perception experiment were carried out, the results of which indicate: (1) The negative interpretation of the word ['liya] (few) is produced with focus on the quantifier, whereas the positive one with focus on the preceding verb. (2) The word in focus has an enlarged tonal range associated with the stressed syllable, while the material out of focus is tonally compressed. (3) The stressed syllable of the word in focus, especially the vowel, is significantly longer. (4) The affirmative and negative functions of the quantifier had high identification rates, indicating a one-to-one production and perception relation.
\end{abstract}

Key words: prosody, intonation, quantifiers, Greek.

\section{Introduction}

This paper reports on an experimental study of prosodic production and perception of semantic specification with reference to sentence quantifiers in Greek. The word ['liyo] (Sing.) and ['liya] (Pl.) may have a positive or a negative meaning, which is determined by prosodic structure. E.g. the sentence ['exume 'liya 'xrimata] may mean either "we have some money" or "we have little money". We assume that a sentence with this quantifier may have different interpretations, which are determined by different focus applications. Focus is mostly associated with semantic weighting and information structure. The prosodic structure of focus with regards to the distinction new-old or the most important information unit in a context has been widely studied in many languages (e.g. Botinis 1989, Bruce1977, Gussenhoven 2008), while in a recent study (Botinis et al. 2011), focus has been reported for the communicative distinction of agreement-disagreement.

\section{Experimental methodology}

In accordance with one production experiment, the prosodic structure of the word ['liya] with distinct semantic specification was examined, i.e. positive or negative, in the context of the carrier sentence [mu 'minane_le' mona] (I have a few lemons left). Ten female speakers, in their early twenties, with standard Athenian pronunciation, produced the above speech material 2 times (normal tempo). The experimental sentences were answers of the leading question [ $\theta \mathrm{a}$ mu 'fcaksis mna lemo'naða] "Will you make me a lemonade?". The speakers were instructed to answer either positively or

ExLing 2011: Proceedings of 4th Tutorial and Research Workshop on Experimental Linguistics, 25-27 May, Paris, France 
negatively. The recordings took place at Athens University Laboratory of Phonetics and Computational Linguistics. The speech material was analysed with Praat, and measurements were taken for each segment duration of the word ['liya] as well as $2 \mathrm{~F} 0$ points per syllable for each utterance.

In accordance with one perception experiment, 10 productions for each interpretation (negative/positive) were presented in random order to 20 Greek native speakers (10 males, 10 females), who were instructed to categorise each stimulus as semantically negative or positive.

\section{Results}

The results of the production experiment are shown in Figures 1-3.
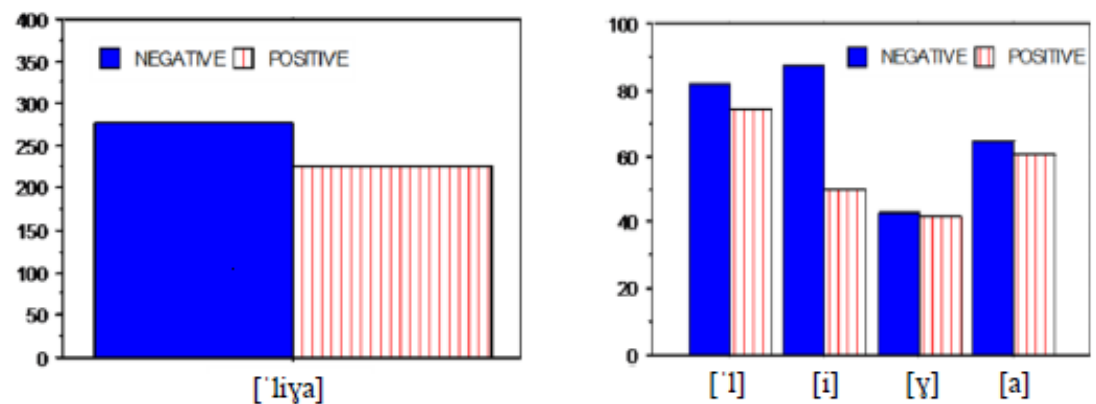

Figure 1. Word duration (ms) of the Figure 2. Segmental durations (ms) of quantifier with negative (focus) and the quantifier with negative (focus) positive (no focus) interpretation. and positive (no focus) interpretation.

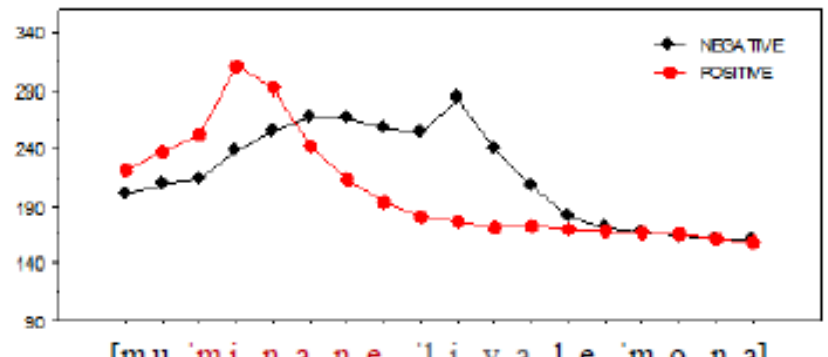

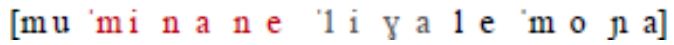

Figure 3. Mean F0 measurements (Hz) with negative semantic interpretation (quantifier ['liya] in focus) and positive one (verb ['miname] in focus).

The average word duration (Fig.1) of the quantifier when in focus (negative interpretation) is $277 \mathrm{~ms}$, and out of focus (positive interpretation) $226 \mathrm{~ms}$. Focus has a lengthening effect of $50 \mathrm{~ms}(18,3 \%)$; this difference though does not reach the significance level of $0.05(\mathrm{t}(\mathrm{df} 30)=0,45, \mathrm{p}=0,65)$. The consonant [1] in focus (Fig.2) is $81 \mathrm{~ms}$ and out of focus $74 \mathrm{~ms}(7,7 \mathrm{~ms}$, $9,4 \%$ difference). This difference is not statistically significant at 0.05 level 
$(\mathrm{p}=0.15)$. The duration of the vowel [i] in focus is $87 \mathrm{~ms}$ and out of focus 50 $\mathrm{ms} \quad(37,1 \mathrm{~ms}, 42 \%$ difference); this difference is highly significant $(\mathrm{p}<0,0001)$. Neither the consonant $[\gamma]$ nor the vowel [a] show any worth mentioning difference in focus and out of focus and, thus, focus application has no effect on unstressed syllables. The mean duration for the first syllable ['li] when in focus is $169 \mathrm{~ms}$, and out of focus $124 \mathrm{~ms}(44,8 \mathrm{~ms}, 26,5 \%$ difference); this difference between the two interpretations for the first syllable is highly significant $(\mathrm{t}(\mathrm{df} 30)=3,90, \mathrm{p}<0,0005)$. Neither the unstressed syllable [ya] nor the syllabic constituency segment show any worth mentioning difference.

With regards to intonation (Fig.3), 2 very distinct tonal structures are observed: (a) with focus on the quantifier for the negative interpretation, and (b) with focus on the verb for the positive interpretation. In rendition (a), a prominent rise-fall tonal movement is produced, with its peak aligned with the vowel of the stressed syllable of the quantifier ['liya] in focus. In rendition (b), a prominent rise-fall movement is also produced, with its peak aligned with the vowel of the stressed syllable of the verb ['minane] in focus. In both cases, the post-focal part of the utterance is tonally compressed, highlighting the respective word in focus. The tonal distinction is statistically significant $(\mathrm{F}(1,574)=9,28, \mathrm{p}=0.002)$.

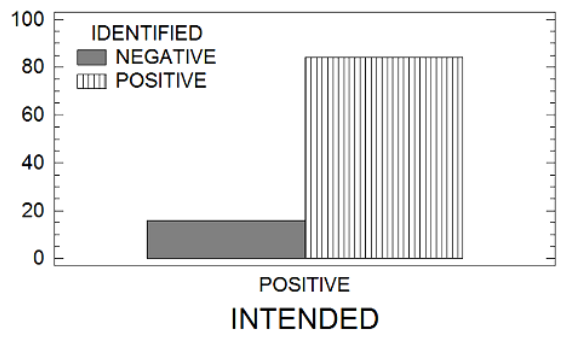

Figure 4. Identification (\%) for intended positive interpretations.

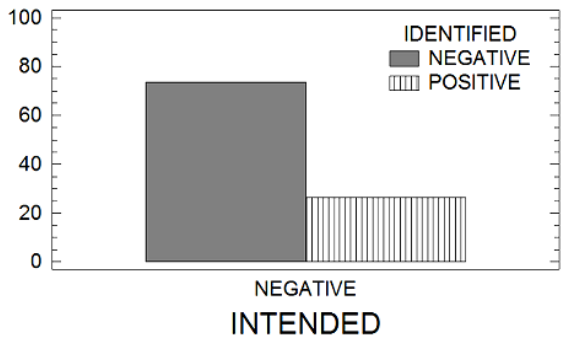

Figure 5. Identification (\%) for intended negative interpretations.

The results of the perception experiment are shown in Figures 4-5. The positive interpretation (Fig.4) was identified as intended $84 \%$ of times, and the negative interpretation (Fig.5) $73 \%$ of times. The identification results in total were statistically significant $\left(\mathrm{x}^{2}(\mathrm{df} 1)=131,41, \mathrm{p}<0.01,99 \%\right.$ conf. level $)$.

\section{Discussion}

Focus is a complex linguistic concept, which has been studied from many different perspectives. With reference to phonetic studies, several local and global correlates have been reported, such as local tonal expansion, local tonal alignment and global compression, especially a postfocal tonal 
flattening (e.g. Botinis 1989, Hirst, Di Cristo 1998, Xu et al. 2005). According to the results of this study, the typical tonal structure of a tonal expansion aligned with the stressed syllable of the word in focus, in combination with a global tonal compression, especially a post-focal one, has been corroborated. However, the segmental material of the stressed syllable of the word in focus, especially the vowel, has a substantial lengthening effect. Given that this effect has not been constantly reported in previous studies for Greek, we may assume that duration structure is a variable parameter of focus, whereas tonal structure is a constant one. In fact, in the present study, duration was found to be a significant prosodic correlate of focus with a quantification distinction.

In conclusion, this study highlights that focus prosodic structure has a distinctive function for quantification with regards to sentence affirmation and negation in Greek. These distinctions were clearly perceived by listeners, indicating a one-to-one production and perception relation.

Therefore, we might assume a one-to-many relation between focus prosodic structure and semantic interpretation. According to this hypothesis, focus production may have only one prosodic structure, which may be related to several interpretations. Apparently, information focus reported in several studies of Greek prosody (e.g. Botinis 1989) and semantic specification of quantification reported in this paper do share the basic prosodic characteristics, at least tonal ones. However, focus application on the quantifier under examination has a sentence negation function rather than a highlighting one, which puts new dimensions to current prosodic knowledge and linguistic theory in general.

\section{Acknowledgements}

This research is supported by the University of Athens "Kapodistrias" project. Thanks to the participants of the experiments, to Marios Fourakis for his feedback, and to Sonia Loui for her help in the perception experiment.

\section{References}

Botinis, A. 1989. Stress and Prosodic Structure in Greek. Lund University Press.

Botinis, A. Bakakou-Orphanou, A., Chaida, A. 2011 (to appear). Sentence functions and tonal semantics in Greek. $7^{\text {th }}$ AISV National Conference, Lecce, Italy.

Bruce, G. 1977. Swedish Word Accents in Sentence Perspective. Lund, Gleruup.

Hirst, D., Di Cristo, A. (eds). 1998. Intonation systems: a survey of twenty languages. Cambridge University Press.

Gussenhoven, C. 2008. Notions and subnotions in information structure. Acta Linguistica Hungarica 55, 381-395.

$\mathrm{Xu}$, Y., Xu, C.X. 2005. Phonetic realization of focus in English declarative intonation. Journal of Phonetics 33, 159-197. 